\title{
Incorporating polysomnography into obstructive sleep apnoea phenotyping: moving towards personalised medicine for OSA
}

\author{
Jean Louis Pépin, ${ }^{1,2}$ Sebastien Bailly, ${ }^{1,2}$ Renaud Tamisier ${ }^{1,2}$
}

Obstructive sleep apnoea (OSA) is a major health concern worldwide with multiorgan consequences and the resulting increased economic and social burden. ${ }^{1}$ OSA is associated with obesity in more than $60 \%$ of cases and often with comorbidities including hypertension, arrhythmia, stroke, coronary heart disease and metabolic dysfunction. The comorbidities are of major importance because they have a significant impact on healthcare use and mortality in patients with OSA. Only half of the patients with OSA are symptomatic and a significant percentage of subjects are referred and treated with the goal of limiting their cardiometabolic risk. This heterogeneity in a highly prevalent chronic disease with millions of patients on home-based longterm treatment requires the complete reshaping of patient characterisation and therapeutic strategies. $^{2}$

Detailed phenotyping is the prerequisite for the development of precision and personalised medicine in chronic disease and particularly in OSA. ${ }^{3}$ The phenotyping of patients with OSA has been tackled in several different ways. One strategy has been to address the anatomical and physiological traits underlying OSA occurrence and the range of severity. ${ }^{4}$ The pathophysiological causes of OSA vary considerably between patients. Determining the respective contributions of upper airway anatomy and collapsibility, arousal threshold and loop gain (ventilator drive) will help to predict which therapeutic modality would be beneficial to the appropriately targeted patient subgroups. ${ }^{4}$ Another topic has been to define distinct OSA clinical phenotypes. Several recent studies exploiting large data sets have used unsupervised cluster analysis to identify distinct phenotypes. ${ }^{5}{ }^{6}$ Subgroups have

\footnotetext{
1 Institut National de la Santé et de la Recherche Médicale (INSERM), U 1042, HP2 Laboratory (Hypoxia: Pathophysiology), University of Grenoble-Alpes, Grenoble, France

${ }^{2}$ Thorax and Vessels Division, Grenoble University Hospital, Grenoble, France
}

Correspondence to Professor Jean Louis Pépin, Laboratoire HP2, Inserm 1042, Universite Grenoble Alpes, Grenoble 38400, France; jpepin@chu-grenoble.fr been recognised that varied considerably in age, gender, symptoms, obesity, comorbidities and environmental risk factors. ${ }^{5}$ The most significant differences between clusters were minimally symptomatic versus sleepy patients with OSA, lean versus obese, and among obese patients different combinations of comorbidities and environmental risk factors. The next priority was to evaluate the time course of fluctuations in these clusters and how these phenotypes are predictors of outcomes. ${ }^{6}$ The longitudinal association between OSA clusters and continuous positive airway pressure (CPAP) adherence was first addressed by Gagnadoux et $a l^{6}$ with reduced CPAP compliance in situations of an association between insomnia and OSA, or mild OSA and comorbid OSA. In the study of Zinchuck et al, ${ }^{7}$ the percentage of patients with optimal CPAP adherence ( $>4$ hours per night) varied broadly among clusters from $25 \%$ to $50 \%$. The novelty here was to demonstrate in the whole study population that regular CPAP use was associated with a $36 \%$ decreased risk of primary outcome, but this attenuation varied by cluster. The impact of CPAP was significant only in the 'periodic limb movements of sleep (PLMS)' and 'hypopnoea+hypoxia' clusters. This suggests that improvement of sleep and the suppression of hypoxia are of equivalent importance to improve prognosis. In the entire population of 72217 CPAP-treated patients in Catalonia, specific profiles of patient with OSA defined by cluster analysis were associated with different mortalities and healthcare resource uses. ${ }^{8}$

The majority of previous OSA clustering analyses have included a limited number of indices from sleep studies characterising sleep architecture and quality. Information was frequently restricted to the apnoea +hypopnoeaindex and markers of intermittent hypoxia severity. In a cross-sectional, multisite observational US Veteran cohort $(\mathrm{n}=1247)$, Zinchuck et $a l^{7}$ performed a principal components-based clustering analysis including various polysomnographic features allowing a better coverage of the sleep architecture/arousals and sleep disorders associated with OSA. Cox survival analysis was used to evaluate longitudinal relationships between discovered clusters and the combined outcome of incident transient ischaemic attack, stroke, acute coronary syndrome or death. Zinchuck et $a l^{7}$ not only nicely decipher new polysomnographic OSA phenotypes but also report on their cardiovascular implications in OSA. The novelty of these results is to underline the crucial importance of alterations in sleep duration or quality and associated sleep disorders in terms of cardiovascular outcomes and death. The worse prognosis was observed in the phenotypes with poor sleep/sleep fragmentation or PLMS. Owing to the high prevalence of the main sleep disorders (eg, insomnia, PLMS, chronic sleep deprivation), it is expected that a combination of sleep problems would be seen frequently in routine clinical practice of patients referred for OSA care. A prevalence of insomnia symptoms of up to $60 \%{ }^{9}$ has been reported in patients with OSA, and between $30 \%$ and $67 \%$ of patients with insomnia have an apnoea + hypopnoeaindex greater than five per hour. ${ }^{10}$ The coexistence of insomnia has been shown to negatively affect CPAP compliance in some studies. Also an association between OSA and PLMS was found in more than $40 \%$ of patients. ${ }^{11}$

The intermediary mechanisms triggering cardiovascular sleep apnoea comorbidities are now well described. The repetitive occurrence of partial or complete pharyngeal collapses during OSA induces chronic intermittent hypoxia with microarousals ending the events that both subsequently result in low-grade inflammation, sympathetic overactivity and oxidative stress. ${ }^{12}$ Increase on sympathetic tone, low-grade inflammation, oxidative stress and endothelial dysfunction are also key features linking other sleep disorders and cardiovascular diseases. ${ }^{13}$ Zinchuck et $a l^{7}$ clearly suggest that the cumulative impact of two sleep disorders or sleep problems is associated with a greater risk of cardiovascular events and death. Accordingly, a positive association between PLMS and cardiovascular events and/or mortality has been recently demonstrated in a systematic review. ${ }^{14}$ In line with the data of Zinchuck et al, ${ }^{7}$ a greater risk of death was attributed to PLMS as it more systematically induces microarousals.

Rapid eye movement (REM) sleep especially phasic REM sleep is associated with higher sympathetic activity and cardiovascular instability both in healthy individuals and in patients with OSA. ${ }^{15}$ Recent studies have indicated that predominant REM OSA is independently associated with prevalent and incident hypertension. ${ }^{16}$ The 'REM and hypoxia' cluster in the Zinchuck et al's ${ }^{7}$ paper was associated with higher (unadjusted) 


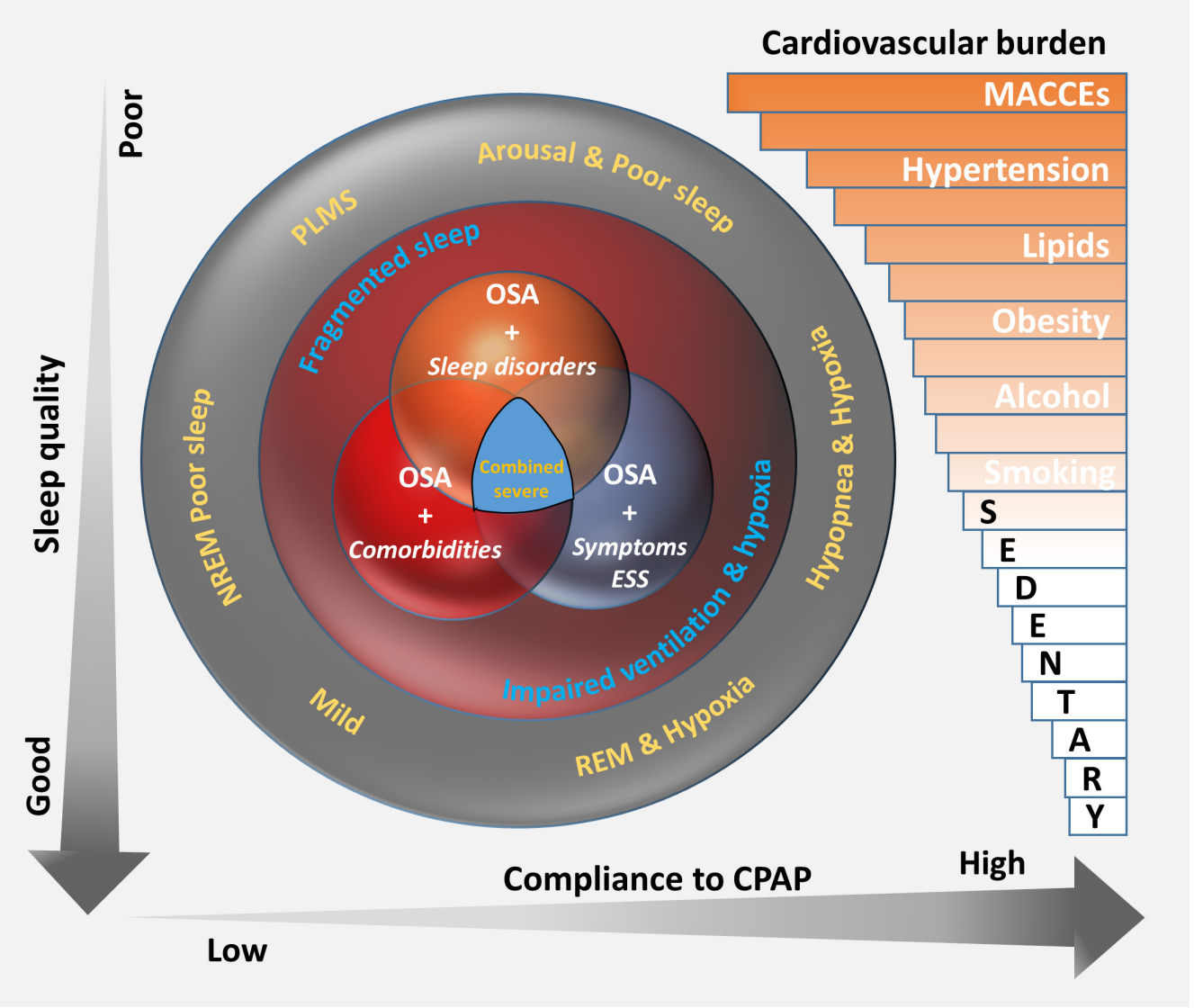

Figure 1 Clusters of clinical and polysomnography phenotypes in OSA and their impacts on outcomes. CPAP, continuous positive airway pressure; ESS, Epworth Sleepiness Scale; MACCEs, major adverse cardiac and cerebrovascular events; NREM, non-rapid eye movement; OSA, obstructive sleep apnoea; PLMS, periodic limb movements of sleep; REM, rapid eye movement.

prevalence of hypertension but not with increased incidence of cardiovascular events or death. Actually, contemporary data from the Sleep Heart and Health Study show that severe OSA present only during REM sleep is associated with cardiovascular events only in people who have prevalent cardiovascular disease. ${ }^{17}$ Also smaller percentages of slow wave sleep $(\mathrm{N} 3+\mathrm{N} 4)$ are predictive of incident hypertension independent of potential confounders, including indices of sleep apnoea and sleep fragmentation. ${ }^{18}$ All these data including the Zinchuck et al's. ${ }^{7}$ contribution tell us that objective indices of sleep microstructure and macrostructure are probably required for optimal characterisation, risk stratification and treatment strategies for patients with OSA. There is a strong contradiction between this statement and the continuous efforts of payers favouring the replacement of polysomnography by home sleep testing mainly using respiratory polygraphy. The largest study to date in the field ${ }^{19}$ suggests that in patients referred with intermediate-to-high suspicion of sleep apnoea, home respiratory polygraphy management is non-inferior to polysomnography, with a substantially lower cost. Midterm outcomes were the same for the
Epworth Sleepiness Scale at 6 months, quality-of-life measures, blood pressure or CPAP adherence. In contrast, the data of Zinchuck et $\mathrm{al}^{7}$ raise the question of whether the lack of sleep recording and information on sleep architecture will affect long-term patient outcomes. A home sleep apnoea test remains recommended as an alternative to polysomnography for the diagnosis of OSA in uncomplicated adults presenting with signs and symptoms that indicate an increased risk of moderate to severe OSA. ${ }^{20}$ However, the data from Zinchuck et $a l^{7}$ support the American Academy of Sleep Medicine recommendation to stick to polysomnography, rather than home sleep apnoea testing, for the diagnosis of OSA in patients with significant comorbidities or suspicion of associated sleep disorders. The preservation of a good balance between the two diagnostic strategies in appropriate subgroups of OSA is one of the main challenges of personalised medicine in OSA (figure 1).

In summary, this heterogeneity in OSA, which was expressed as differences in OSA phenotypes, challenges the effectiveness of uniform diagnostic and management strategies. Zinchuck et al provide new evidence about the prognostic value of polysomnographic indices for precision medicine in OSA.

Acknowledgements We thank Alison Foote PhD (Grenoble Alps University Hospital) for critically editing the manuscript.

Contributors All authors have been involved in the writing, have a final read and approved the submission of the manuscript.

Funding This work was partly supported by the French National Research Agency in the framework of the "Investissements d'avenir" program (ANR-15IDEX-02).

Competing interests None declared.

Patient consent Not required.

Provenance and peer review Commissioned; externally peer reviewed.

(C) Article author(s) (or their employer(s) unless otherwise stated in the text of the article) 2018. All rights reserved. No commercial use is permitted unless otherwise expressly granted.

A) Check for updates

To cite Pépin JL, Bailly S, Tamisier R. Thorax 2018:73:409-411.

Accepted 5 February 2018

Published Online First 24 February 2018 


\section{CSLinked}

http://dx.doi.org/10.1136/thoraxjnl-2017-210431

Thorax 2018;73:409-411.

doi:10.1136/thoraxjnl-2017-210943

\section{REFERENCES}

1 Lévy P, Kohler M, McNicholas WT, et al. Obstructive sleep apnoea syndrome. Nat Rev Dis Primers 2015;1:15015.

2 Pépin JL, Tamisier R, Hwang D, et al. Does remote monitoring change OSA management and CPAP adherence? Respirology 2017;22:1508-17.

3 König IR, Fuchs O, Hansen G, et al. What is precision medicine? Eur Respir J 2017;50:1700391.

4 Carberry JC, Amatoury J, Eckert DJ. Personalized management approach for OSA. Chest 2017.

5 Bailly S, Destors M, Grillet Y, et al. Obstructive sleep apnea: a cluster analysis at time of diagnosis. PLOS One 2016;11:e0157318.

6 Gagnadoux F, Le Vaillant M, Paris A, et al. Relationship between OSA clinical phenotypes and CPAP treatment outcomes. Chest 2016;149:288-90.

7 Zinchuk AV, Jeon S, Koo BB, et al. Polysomnographic phenotypes and their cardiovascular implications in obstructive sleep apnoea. Thorax 2018;73:472-80.
8 Turino C, Bertran S, Gavaldá R, et al. Characterization of the CPAP-treated patient population in Catalonia. PLoS One 2017;12:e0185191.

9 Pepin JL, Gagnadoux F, Foote A, et al. Combination of obstructive sleep apnoea and insomnia treated by continuous positive airway pressure with the SensAwake pressure relief technology to assist sleep: a randomised cross-over trial protocol. BMJ Open 2017:7:e015836.

10 Luyster FS, Buysse DJ, Strollo PJ. Comorbid insomnia and obstructive sleep apnea: challenges for clinical practice and research. J Clin Sleep Med 2010;6:196-204.

11 Al-Alawi A, Mulgrew A, Tench E, et al. Prevalence, risk factors and impact on daytime sleepiness and hypertension of periodic leg movements with arousals in patients with obstructive sleep apnea. J Clin Sleep Med 2006;2:281-7.

12 Arnaud C, Beguin PC, Lantuejoul S, et al. The inflammatory preatherosclerotic remodeling induced by intermittent hypoxia is attenuated by RANTES/CCL5 inhibition. Am J Respir Crit Care Med 2011;184:724-31

13 Pepin JL, Borel AL, Tamisier R, et al. Hypertension and sleep: overview of a tight relationship. Sleep Med Rev 2014;18:509-19.

14 Kendzerska T, Kamra M, Murray BJ, et al. Incident cardiovascular events and death in individuals with restless legs syndrome or periodic limb movements in sleep: a systematic review. Sleep 2017;40.

15 Alzoubaidi M, Mokhlesi B. Obstructive sleep apnea during rapid eye movement sleep: clinical relevance and therapeutic implications. Curr Opin Pulm Med 2016;22:545-54.

16 Mokhlesi B, Finn LA, Hagen EW, et al. Obstructive sleep apnea during REM sleep and hypertension. results of the Wisconsin Sleep Cohort. Am J Respir Crit Care Med 2014;190:1158-67.

17 Aurora RN, Crainiceanu C, Gottlieb DJ, et al. Obstructive sleep apnea during rapid eye movement sleep and cardiovascular disease. Am J Respir Crit Care Med 2018;197:653-60.

18 Javaheri S, Zhao YY, Punjabi NM, et al. Slow-wave sleep is associated with incident hypertension: the sleep heart health study. Sleep 2017;41.

19 Corral J, Sánchez-Quiroga MÁ, Carmona-Bernal $C$, et al. Conventional polysomnography is not necessary for the management of most patients with suspected obstructive sleep apnea. noninferiority, randomized controlled trial. Am J Respir Crit Care Med 2017:196:1181-90.

20 Rosen IM, Kirsch DB, Chervin RD, et al. Clinical use of a home sleep apnea test: an american academy of sleep medicine position statement. J Clin Sleep Med 2017;13:1205-7. 